This is the author's version of a work that was accepted for publication in the Journal of Crystal Growth. Changes resulting from the publishing process, such as peer review, editing, corrections, structural formatting and other quality control mechanisms may not be reflected in this document. Changes may have been made to this work since it was submitted for publication. A definitive version was subsequently published in Journal of Crystal Growth, Volume 402, 15 September 2014, Pages 215-221.

http://doi.org/10.1016/j.jcrysgro.2014.06.027 


\title{
Investigation of Growth Rate Dispersion in Lactose Crystallisation by AFM Dincer $T D^{1}$, Ogden $M I^{2}$ and Parkinson G $\mathbf{M}^{2}$
}

\author{
${ }^{1}$ Food Science and Technology Program, School of Public Health, ${ }^{2}$ Department of Chemistry \\ Curtin University, GPO Box U1987, Perth WA 6845, Australia
}

\begin{abstract}
$\alpha$-Lactose monohydrate crystals have been reported to exhibit growth rate dispersion (GRD). Variation in surface dislocations has been suggested as the cause of GRD, but this has not been further investigated to date. In this study, growth rate dispersion and the change in morphology were investigated in situ and via bottle roller experiments. The surfaces of the (010) faces of crystals were examined with Atomic Force Microscopy. Smaller, slow growing crystals tend to have smaller (010) faces with narrow bases and displayed a single double spiral in the centre of the crystal with $2 \mathrm{~nm}$ high steps. Additional double spirals in other crystals resulted in faster growth rates. Large, fast growing crystals were observed to have larger (010) faces with fast growth in both the $\mathbf{a}$ and $\mathbf{b}$ directions (giving a broader crystal base) with macro steps parallel to the (c direction). The number and location of spirals or existence of macro steps appears to influence the crystal morphology, growth rates and growth rate dispersion in lactose crystals.
\end{abstract}

*Corresponding author, Phone: +61 8 9266 7497, e-mail: T.Dincer@.curtin.edu.au

Keywords: A1.Optical Microscopy, A1. Atomic Force Microscopy, A2.Growth from solution, A2.Single crystal growth, B1.Organic Compounds 


\section{Introduction}

White and Wright [1] first reported a spread of an initially narrow sized crystal population in sucrose, which could not be explained by size dependent growth. They used the term growth rate dispersion (GRD) to describe the variation in growth rate among crystals of the same size while they grow under constant macroscopic conditions and it is still not understood well [2]. This causes widening of the crystal size distribution and is a significant factor in the control of the crystal size distribution (CSD) in batch crystallisers. GRD has a significant influence on particle size distribution of the crystalline product in industrial crystallisers especially in the low size range. The consequence of GRD is an increase in the numbers of small slow growing crystals and therefore a reduction of the mean particle size in both batch and continuous crystallisers [3]. Since then, many other crystals have been reported to exhibit growth rate dispersion, for example potash alum [3] , citric acid [4], fructose [5], sodium chloride [6] and others [7]. It has been reported for crystals generated by both primary and secondary nucleation [8].

Possible causes of GRD are the specific surface structure of the growing crystal [9], varying degrees of strain in the crystal lattice [3,10,11], random surface adsorption [12] or physical incorporation of impurities, and fluctuation in supersaturation [13] . The nuclei growth rates were reported to be strain controlled at high growing supersaturation and dislocation controlled at low growing supersaturation $[14,15]$. The surface integration step for crystal growth is thought to be the primary factor in mechanisms of growth rate distribution, and the Burton-Cabrera-Frank (BCF) [16] growth theory is often used to provide qualitative explanation of the growth rate dispersion phenomena. From the BCF theory, the growth rate of a crystal face is dependent on the number, sign and location of screw dislocations emerging at the surface of a growing crystal. A distribution of growth rates is a result of the varying dislocation networks and densities among nuclei and seed crystals [17]. 
Bennema stated that occasionally the growth rate of a certain face may vary by a factor of 1 to 5 for 1 to 5 collaborating spirals or may become zero below a certain supersaturation for the case of spirals of opposite sign or where dislocations are absent [18]. Secondary nuclei of sodium chloride crystals were reported to exhibit higher dispersion at smaller size together with zero growth rates [19]. This was explained to be the consequence of stress induced reduction of growth rates being dominant at smaller size. Gradual increase of size reduces the strain and contribution of dislocation becomes dominant. Recently, Pantaraks et al [20] reported that GRD is a result of the difference in microscopic surface roughness caused by surface nuclei generated on the surface of crystals. The growth history of crystals was found to have a significant effect on the future crystal growth rate. For example, a crystal grown in a high supersaturation environment was found to grow at a slower rate when moved to a lower supersaturation environment compared to crystals grown at low supersaturation over the same time.

The presence of GRD in lactose crystallisation was first reported by Visser [21] and he recommended further research, for instance in a growth cell. There have been three published works on the GRD in lactose crystallisation. Shi et al [22] reported that each crystal grows at its inherent constant rate but different crystals have different growth rates (CCG Model) in contact nucleation experiments. Liang et al [23] studied the effect of growth rate dispersion on lactose crystal size distribution from a continuous cooling crystalliser. A larger number of particles of smaller sizes than predicted by the standard population balance model were present and the semi-logarithmic population density versus crystal size plot showed a curvature at small sizes (less than $40 \mu \mathrm{m}$ ). They determined that the linear extrapolation technique used to calculate the growth rate in the cooling crystalliser overestimates actual growth rates by neglecting the high number of slow growing crystals. The crystal size distribution was successfully modelled using a two-component rate distribution indicating the existence of two distinct types of nuclei. These were classed as fast growers (product crystals) 
and slow growers (fines). The average ratio of fast growers to slow growers was found to be 2.2 for lactose and 3 for sucrose by Liang and Hartel [24]. Dincer et al reported GRD in a seeded system and found that the four main faces of lactose crystal displayed GRD and it increased with increasing supersaturation and temperature [25].

The crystal structure of a-lactose monohydrate crystals has been reported [26]. The unit cell contains two lactose and two water molecules. The unit cell dimensions are a=7.982 $\AA$, $\mathrm{b}=21.562 \AA$, c $=4.824 \AA$ and $\beta=109.57^{\circ}$ (Monoclinic, $\mathrm{P} 2{ }_{1}$ ). An important characteristic of $\alpha$ lactose monohydrate crystals grown from aqueous solutions is an unequal development of opposing faces (i.e. the (hkl) and ( $\bar{h} \bar{k} \bar{l})$ faces); in particular, the (110), (110) and (010) faces are significantly larger than the $(\overline{1} \overline{1} 0),(1 \overline{1} 0)$ and the $(0 \overline{1} 0)$ faces, respectively, with the last face missing in many crystals. The crystal morphology is dominated by the $(0 \overline{1} \overline{1})$, $(0 \overline{1} 1)$ and (010) faces. Large (100) and (100) faces are also present. The resulting crystal is tomahawk-shaped as shown in Figure 1. Crystals of $\alpha$-lactose monohydrate grow mainly in the $+b$ direction. Van Krevald and Michaels [27] and later Visser [28] stated that the tomahawk shaped morphology of $\alpha$-lactose monohydrate is caused by selective blocking of the $(0 \overline{1} 0),(0 \overline{1} 1)$ and $(0 \overline{1} \overline{1})$ faces by $\beta$-lactose molecules in the solution. Because both $\alpha$ and $\beta$ lactose have the same galactosyl moiety, the $\alpha$-lactose molecules of the crystal might form bonds as easily with the galactose moiety of a $\beta$ molecule as that of an $\alpha$ molecule. In the tomahawk, the $\alpha$ molecules are orientated along the $b$ axis, with all the glucose moieties pointing in the $-b$ direction. Once integrated, a $\beta$ molecule would inhibit further growth of $\alpha$ lactose at that growth site. This was proved by Raghavan et al by analysing the $\beta$-lactose content of different sections of large lactose crystals [29]. The $\beta$-lactose content of the sections on the $\{0 \overline{1} \overline{1}\}$ faces of the crystal were found to be higher than other sections which resulted in higher strain on these faces as shown by X-Ray tomography. 
In the absence of impurities, the size of the face is inversely related to the growth rate of the face. Fast growing faces are usually the smallest or grow out of existence. The morphology of crystals is determined by the slowest growing faces.

We have previously reported in situ growth rates of single $\alpha$-lactose monohydrate crystals [25]. In these experiments, growth was followed for 3-4 hours and no change in morphology was observed over this time. Following this publication, longer in situ runs were investigated and in one of the experiments in which crystals were grown for four days $\left(30^{\circ} \mathrm{C}\right.$ and $40 \mathrm{~g}$ lactose $/ 100 \mathrm{~g}$ water concentration), the crystals with broader base (a direction) grew faster than all the other crystals with narrower bases and the none of the crystals with narrow bases developed broader bases (Figure 2). This observation prompted further investigation on morphological differences between slow and fast growing lactose crystals using atomic force microscopy (AFM), in an effort to understand what the differences between slow and fast growing crystals are.

In this research, the growth rate dispersion of lactose crystals and changes in crystal morphology were investigated in an in situ cell (stagnant) and in bottle roller (dynamic) conditions. For AFM investigation, measuring the growth rate of individual crystals and investigating the (010) face of the same crystals proved to be impractical. Instead crystals were grown in conditions which minimise secondary nucleation, breakage or agglomeration for 10 days to reach sizes which allows manual manipulation of crystals for imaging. The (010) faces of lactose crystals with different sizes were imaged and the relationship between surface structure, morphology and the growth rates were investigated.

\section{Experimental Method}

$\alpha$-Lactose monohydrate powder (L-8783) was purchased from Sigma Chemicals. All seed preparations and growth experiments were performed at $30{ }^{\circ} \mathrm{C}$, and lactose concentration 
of $40 \mathrm{~g} \alpha$-lactose monohydrate in $100 \mathrm{~g}$ DI water. The supersaturation ratio of $\alpha$-lactose, $\mathrm{s}$, is calculated using equation given by Visser [30]:

$$
s=\frac{C}{C_{s}-F K_{M}\left(C-C_{s}\right)}
$$

$\mathrm{C}$ is total lactose concentration and $\mathrm{C}_{\mathrm{S}}$ is final solubility of lactose in g anhydrous lactose/100 g water, F, a temperature dependent factor for depression of solubility of $\alpha$-lactose by $\beta$-lactose and $\mathrm{K}_{\mathrm{M}}, \beta / \alpha$ ratio of lactose in mutarotation equilibrium at the relevant temperature.

At this concentration, the relative lactose supersaturation (s-1) was calculated to be 0.55 .

In situ crystal growth experiments were performed to measure the growth rates of individual crystals using an apparatus developed by Reyhani and Parkinson [31] that consists of a Transmission type Optical Microscope, an in situ cell, a Grant W14 (Grant Instruments Ltd.) circulating water bath, Pulnix TM-9701 Camera (Progressive Scanning Full Frame Shutter Camera), and a Pentium II Computer. The in situ cell has two compartments: sample (upper) and circulating water for temperature control (lower) separated by circular thin glass cover slips. The volume of the sample compartment is $5 \mathrm{ml}$. This set up allows in situ observation of crystal growth rates and morphology at constant temperature and supersaturation. Lactose seed crystals were prepared by dissolving (with heating) $40 \mathrm{~g} \alpha$ lactose monohydrate in DI $100 \mathrm{~g}$ water to $70{ }^{\circ} \mathrm{C}$. After cooling to room temperature, the solution was filtered through a $0.45 \mu \mathrm{m}$ filter membrane and placed in an ultrasonic water bath (Elma Transsonic T570 $20 \mathrm{kHz}$ ) for three minutes to initiate nucleation at the same time. The quiescent solution was then covered and kept in a water bath at $30{ }^{\circ} \mathrm{C}$ for 24 hours, allowing seed crystals to grow. Fresh lactose solution along with a few drops of seed stock suspension were transferred into the cell and images of crystals were ecorded over time, for durations between 3-6 hours. The length of crystals in the b direction from the tip of the 
crystal (L) and the width of the base of the in the a direction (W) were measured using the OPTIMAS Software (Adept Electronic Solutions). The growth rate of the (010) face was measured by calculating the slope of the length versus time plot. As the $(0 \overline{1} 0)$ face does not grow in aqueous solution [27] (the length was measured from the tip of the crystal, the growth of adjacent faces is not measured), the calculated rate of increase in length by time is the growth rate of the (010) face. A total of 227 crystals were investigated in five experiments. Initial crystal size is the crystal size at the beginning of experiments which is after 24 hours after initiation of nucleation. Average values with standard deviation were calculated.

To follow the growth for longer duration of growth, seed crystals were grown in dynamic conditions. Nalgene bottles $(100 \mathrm{ml})$ were filled with lactose solution and $1 \mathrm{ml}$ of seed stock solution prepared using the same method. Bottles were placed in a bottle roller gently (3 rpm) rotating at $30{ }^{\circ} \mathrm{C}$ and were removed after 1, 2, 3, 6 and 7 days ( filtered through $0.45 \mu \mathrm{m}$ filter membrane). For each sample, the length (L) in the $\mathbf{b}$ direction and width (W) of the base of the crystal in the a direction of lactose crystals were measured and the crystal size distribution was calculated (100-200 crystals). The average growth rate of the (010) face was calculated by dividing the crystal dimension (L) with duration of growth. The $\beta$-lactose content of crystals from day 1-7 and also small and large crystals in the day 7 batch were measured by GC [32]. GC samples were prepared and were derivatized in pyridine with TMSIM (N-trimethylsilylimidazole) [32] A Hewlett Packard 5890A Gas Chromatograph with a non-polar capillary column (BP 5) and a FID detector was used [33].

To investigate the surface of the (010) faces by AFM, crystals large enough to be handled needed to be grown. Crystals were grown in static condition for 10 days at the same supersaturation and temperature. The solution was filtered, crystals were washed with saturated water and water-ethanol mixtures (90, 50, $20 \%$ water and finally saturated ethanol) and dried in an oven at $60^{\circ} \mathrm{C}$ overnight. The crystal size in the $\mathbf{b}(\mathrm{L})$ and the $\mathbf{a}(\mathrm{W})$ directions 
were measured. Growth rate was estimated by dividing the crystal dimension with the duration of growth. A total of 11 crystals corresponding to $0.1,0.2$ and $0.3 \mu \mathrm{m} / \mathrm{min}$ growth rates were handpicked and mounted on to a removable adhesive (Bostic, Blu tack) to image the (010) face of the crystal. Images were obtained using a Digital Instrument Nanoscope E AFM, operated in the contact mode, in air, using $\mathrm{Si}_{3} \mathrm{~N}_{4} 200 \mu$ triangular tip cantilevers with a spring constant of $0.12 \mathrm{~N} / \mathrm{m}$. All the images were collected in the height mode. Locations of spirals were marked on the optical images taken of the same crystal.

\section{Results and Discussion}

The secondary nucleation threshold (SNT) at $30{ }^{\circ} \mathrm{C}$ is $35.87 \mathrm{~g}$ anhydrous lactose $/ 100 \mathrm{~g}$ water which is equivalent to $38.50 \mathrm{~g} \alpha$-lactose monohydrate/100 g water [34]. Lactose concentration used in this work was $40 \mathrm{~g}$ $\alpha$-lactose monohydrate/100g water which is 1.4 $\mathrm{g} / 100 \mathrm{~g}$ above the SNT, therefore the formation of secondary nuclei is possible. In relative supersaturation terms, this exceeds the metastable limit by a modest 0.06 and hence by growing crystals in a stagnant environment or with a slow rotation, the effect of secondary nucleation was minimized, but a small amount of secondary nucleation was expected.

Results of the in situ growth experiments in which growth rate of individual crystals were measured show that lactose crystals exhibit growth rate dispersion (Figure 3(a)). For example, considering $\sim 50 \mu \mathrm{m}$ sized lactose crystals, growth rates ranged between 0.01 to 0.04 $\mu \mathrm{m} / \mathrm{min}$. The average growth rate was $(0.021 \pm 0.007) \mu \mathrm{m} / \mathrm{min}$. No indication of size dependent growth was observed in the size range investigated. Under comparable experimental condition, crystal growth was reported to be integration controlled [35]. The growth rate was calculated to be $(0.015 \pm 0.004) \mu \mathrm{m} / \mathrm{min}$ for the bottle roller experiments from the slope of time versus size plot and $(0.016 \pm 0.007) \mu \mathrm{m} / \mathrm{min}$ for stagnant growth experiments 
by dividing the average crystal size by the duration of growth. The crystal growth rates calculated after 1 day for both the in situ and bottle roller experiments are identical and after which the growth rates slightly decrease which is a possible indication of secondary nucleation (Figure 3(b)). This is also indicated by the increase in standard deviation. Due to the low driving force, the extent of nucleation is small and hence does not result in a significant reduction of average crystal size. At $30{ }^{\circ} \mathrm{C}$ and (s-1) of 0.55 , the average growth rate of the $(010)$ face of lactose crystals is $(0.017 \pm 0.003) \mu \mathrm{m} / \mathrm{min}$, approximately $1 \mu \mathrm{m}$ per hour.

During experiments, crystals grew at a constant rate and no reduction in growth rate was observed. However, as seed crystals were grown for 24 hours before growth rates were measured, it is not possible to comment if growth rates reduced gradually during earlier stages of growth as suggested by Zekic et al [36].

The change in crystal morphology can be quantified by the ratio of the length in the $\mathbf{b}$ (L) direction to that in the a (W) direction. It was observed that the $\mathrm{L} / \mathrm{W}$ decreases with crystal size, especially above $300 \mu \mathrm{m}$, where it approaches approximately 1.5 (Figure 4(a)). When the $\mathrm{L} / \mathrm{W}$ is plotted against the crystal growth rate of individual crystals, it was observed that high $\mathrm{L} / \mathrm{W}$ ratios are not observed above growth rates of $0.03 \mu \mathrm{m} / \mathrm{min}$. A low $\mathrm{L} / \mathrm{W}$ ratio indicates a broader base, larger (010) face. This is an interesting observation as large crystals which are fast growing crystals displayed fast growth rates not only on the (010) face but also on the (110) and ( $\overline{1} 10)$ faces. Optical imaging of smaller crystals shows that the $(010)$ face seems to be narrow in the a direction with small $\mathrm{W}$, which indicates that the growth rate of the (010) face is faster than the (110) and $(\overline{1} 10)$ faces. Volume diffusion has been reported to be influential only above growth rates of $0.4 \mu \mathrm{m} / \mathrm{min}$ [25] and $0.6 \mu \mathrm{m} / \mathrm{min}$ [37] therefore the changes in morphology cannot be attributed to volume diffusion. 
The $\beta$-lactose content of crystals grown was found to $(2.6 \pm 0.6) \%$ and there was no significant difference in $\beta$-lactose content of crystals grown for different times or between small and large crystals grown for 10 days.

AFM investigation of the (010) faces of lactose crystals was carried out using crystals grown in static conditions for 10 days. The average crystal size was $260 \mu \mathrm{m}$ with an average growth rate of $(0.016 \pm 0.007) \mu \mathrm{m} / \mathrm{min}$. It was not possible to manually handle crystals less than $150 \mu \mathrm{m}$ in size. The minimum crystal size in the sample was $50 \mu \mathrm{m}$ and $20 \%$ of crystals were less than $150 \mu \mathrm{m}$ in size. Crystals were divided into three groups: small: less than 200 $\mu \mathrm{m}$, medium 300-380 $\mu \mathrm{m}$ and large: larger than $380 \mu \mathrm{m}$. This usually corresponded to high, medium and low L/W ratios, respectively, with approximate growth rates of $0.01,0.02$ and $0.03 \mu \mathrm{m} / \mathrm{min}$ which were labelled as slow, average and fast growers. $50 \%$ of crystals were in the medium size range while only $10 \%$ of the crystals were in the large crystal group. While a small amount of secondary nucleation was observed, the small crystals imaged by AFM were unlikely to be secondary nuclei as the smallest AFM-imaged crystal was $150 \mu \mathrm{m}$.

The details of AFM imaged crystals are given in Table 1. The average growth rates of the AFM imaged crystals was inversely correlated with the L/W ratios and directly correlated with the surface area of the (010) face. AFM investigation of these crystals uncovered significant differences in the surface characteristics. Slow growing crystals tended to have only one double spiral in the crystal centre of the (010) face (two spirals on one crystal imaged, S5), where the step height is $2 \mathrm{~nm}$ which corresponds to one unit cell height in the $\mathbf{b}$ direction (two lactose molecules) (Figure 5). The double spiral is not polygonised and has a steeper slope (smaller step spacing) for the steps parallel to the (110) face than the ones parallel to the $(0 \overline{1} \overline{1})$ face. Step spacing on each side of the spirals were found to be different. The average of measurements of four crystals showed spacings of $1040 \mathrm{~nm}$ and $695 \mathrm{~nm}$ for steps parallel to the $(0 \overline{1} \overline{1})$ and $518 \mathrm{~nm}$ and $372 \mathrm{~nm}$ for steps parallel to the (110) face. 
Additional spirals on steps parallel to the $\{0 \overline{1} \overline{1}\}$ face were also observed. The steps parallel to the $\{0 \overline{1} \overline{1}\}$ face are affected by the presence of $\beta$-lactose [28] in the solution and hence these steps are usually rougher than the steps parallel to the (110) face. In particular, the edges of steps closer to the intersection of two steps are affected more.

Crystals exhibiting average growth rates seemed to have multiple spirals in and around the centre of crystals, numbering between 2 and 8 (Figure 6). The step height also starts increasing to 4 and $6 \mathrm{~nm}$. Additional spirals seems to increase the growth rate in the a direction resulting in larger (010) faces.

For the fast growing crystals, the surface is covered by macro-steps parallel to the (110) face of the crystals, and the step height is around 4-10 nm high (Figure 7). The linear growth rate of a face is given by: $G R=V_{\text {step }} h / y_{0}$ where $V_{\text {step }}$ is the velocity of a step, $h$ is the step height and $\mathrm{y}_{\mathrm{o}}$ is the mean step spacing [38]. The linear growth rate of a face increases with increasing step height. Therefore the (010) face will grow faster. The micro-steps on the (010) face are parallel to the steps on the (110) and the $(\overline{1} 10)$ faces (unpublished data). These micro steps may provide continuous flow of growth steps for the (110) and the (110) faces which might increase the growth rate of these two faces. In large crystals, the (110) and ( $\overline{1} 10)$ faces are smaller, sometimes growing out of existence which indicates faster growth rates. For the small and medium size crystals, there appears to be an increase in growth rate with an increasing number of spirals. Larger (010) faces covered with macro steps parallel to (110) are present in the fastest growing crystals.

The number and location of dislocations formed during nucleation and early stages of growth determines the growth rate of individual crystals and the growth rate stays constant. 


\section{Conclusions}

The specific surface structure and growth rate of individual lactose crystals were found to be related, suggesting a link to growth rate dispersion in this system. AFM investigation of the (010) face of small lactose crystals revealed single double spirals in the centre of the $(010)$ face. The step height is $2 \mathrm{~nm}$ high on the spirals observed. The growth rate of the (110) and (110) faces were lower than that of the (010) face, therefore crystals had smaller (010) faces and larger (110) and $(\overline{1} 10)$ faces. Crystals with more dislocations grow faster in the b direction. The position of the additional dislocations determines the morphology of the crystal. Crystals exhibited faster growth rates when additional spirals form on the steps parallel to the c direction, rather than on steps parallel to the a direction. The highest growth rates were observed on crystals which were covered by macrosteps (4-10 nm high) parallel to the c direction. Increase in step height greatly increases the growth rate of the (010) face. Large crystals had lower L/W ratios indicating broader based crystals. In these crystals the (110) and the (110) faces have high growth rates, and they therefore eventually grow out of existence. The difference in growth rates of small and large crystals could be explained by the formation of additional spirals and macro steps in large crystals. No significant difference in $\beta$-lactose contents of slow and fast crystals was found.

In this study, only limited numbers of crystals were examined with AFM but the changes in morphology with growth rate were tested in stagnant and dynamic systems with larger numbers of crystals. While a correlation has been found between surface structures and crystal growth rates, more research is needed to uncover the origins of these structural variations, and hence GRD, in lactose crystallisation. 


\section{Acknowledgements}

This research has been funded by the Dairy Australia and their support and permission to publish this work are gratefully acknowledged. Authors would like to thank Dairy Innovation Australia for financial support to during the preparation of this manuscript.

\section{References}

[1] T.T. White, P.G. Wright, Magnitute of size dispersion effects in crystallisation, Chemical Engineering Progress Symposium series, 67 (1971) 81.

[2] B.M. Misailovic, D.A. Malivuk, A.A. Zekic, M.M. Mitrovic, Non-growing faces of sodium chlorate crystals in supersaturated solution, Crystal Growth \& Design, (2014).

[3] U. Zacher, A. Mersmann, The influence of internal crystal perfection on growth rate dispersion in acontinuous suspension crystalliser, J. Cryst. Growth, 147 (1995) 172-180.

[4] K.A. Berlung, M.A. Larson, Modeling growth rate dispersion of citric acid monohydrate in a continuous crystalliser, AIChE J., 30 (1984) 280-287.

[5] L.D. Shiau, K.A. Gberlung, Growth kinetics of fructose crystals formed by contact nucleation, AIChE J., 33 (1987) 1028-1033.

[6] M.M. Mitrovic, A.A. Zekic, Size distribution of non-growing crystals in supersaturated solutions, J. Cryst. Growth, 2005 (2005) e337-e341.

[7] A.A. Zekic, M.M. Mitrovic, S.M. Elezovic-Hadzic, D.A. Malivuk, Long-time growth rate changes of sodium chlorate, potasiium dihydrogen phosphate and Rochelle salt crystals independent of growth conditions, Industrial and Engineering Chemistry Research, 50 (2011) 8726-8733.

[8] R.J. Judge, E.L. Forsythe, M.L. Pusey, Growth Rate Dispersion In Protein Crystal Growth, Crystal Growth \&Design, 10 (2010) 3164-3168.

[9] L.D. Shiau, The distribution of dislocation activities amongst crystals in sucrose crystallisation, Chemical Engineering Scinece, 203 (2003) 5299-5304.

[10] R.I. Ristic, B.Y. Shekunov, J.N. Sherwood, The influence of synchroton radiation induced starin on the growth and dissolution of brittle and ductile materials, J. Cryst. Growth, 179 (1997) 205-212.

[11] R.I. Ristic, J.N. Sherwood, K. Wojciechowsky, Assesment of strain in samll sodium chloride crystals and its relation to growth rate dispersion, J. Cryst. Growth, 91 (1988) 163.

[12] A.S. Myerson, Handbook of Industrial Crystallisation, Butterworth_Heineman, 1993.

[13] M.R. Singh, D. Ramkrishna, Dispersion in crystal nucleation and growth rates: Implications of fluctuation in supersaturation, Chem. Eng. Sci., 107 (2014) 102-113.

[14] C.M. Jones, M.A. Larson, R.I. Ristic, J.N. Sherwood, The role of dislocation, integral strain and supersaturation on growth rates of sodium nitrate, J. Cryst. Growth, 208 (2000) 520-524.

[15] C.M. Jones, M.A. Larson, Using dislocation and integral strain to model the growth rates of secondary nuclei, Chem. Eng. Sci., 55 (2000) 2563-2570.

[16] W.K. Burton, N. Cabrera, F.C. Frank, Phil. Trans. Roy. Soc. London, 243 (1951) 299.

[17] R.C. Zumstein, R.W. Rousseau, Growth rate dispersion by initial growth rate distribution and growth rate fluctuations, AIChE J., 33 (1987) 121-129. 
[18] P. Bennema, Industrial Crystallization, in: J.W. Mullin (Ed.) Industrial Crystallization, Plenum Press, 1976.

[19] J.N. Sherwood, R.I. Ristic, The influence of mechanical stress on growth and dislocation of crystals, Chem. Eng. Sci., 56 (2001) 2267-2280.

[20] P. Pantaraks, M. Matsuoka, A.D. Flood, Effect of Growth Rate Historyon Current Crystal Growth. 2. Crystal growth of Sucrose, Al(SO4)2.12 H20, KH2PO4 and K2SO4, Crystal Growth and Design, 7 (2007) 2635-2642.

[21] R.A. Visser, Growth of non-ionic lactose at various temperatures and supersaturations, Neth. Milk Dairy J., 36 (1982) 167-193.

[22] Y. Shi, W. Hartel, B. Liang, Formation and growth phenomena of lactose nuclei under contact nucleation conditions, J. Dairy Sci., 72 (1989) 2906-2915.

[23] B. Liang, Y. Shi, R.W. Hartel, Growth rate dispersion effects on lactose crystal size distribution from continuous cooling crystallizer, J. Food Sci., 56 (1991) 848-854.

[24] B. Liang, R.W. Hartel, Techniques for developing nucleation and growth kinetics from MSMPR data for sucrose crystallisation in the presence of growth rate dispersion, J. Cryst. Growth, 108 (1991) 129-142.

[25] T.D. Dincer, M.I. Ogden, G.M. Parkinson, In situ investigation of growth rates and growth rate dispersion of $\alpha$-lactose monohydrate, J. Cryst. Growth, 311 (2009) 1352-1358.

[26] D.C. Fries, S.T. Rao, M. Sundaralingam, Structural Chemistry of carbohydrates III. Crystal and molecular structure of alpha lactose mionohydrate, Acta Crystallographica, B27 (1971) 994.

[27] A. van Kreveld, A. Michaels, Measurement of crystal growth of alpha lactose, J. Dairy Sci., 48 (1965) 259-265.

[28] R.A. Visser, P. Bennema, Interpretation of the morphology of alpha lactose hydrate, Neth. Milk Dairy J., 37 (1983) 109-137.

[29] S.L. Raghavan, R.I. Ristic, D.B. Sheen, J.N. Sherwood, l. Trowbridge, P. York, Morphology of Crystals $\alpha$-lactose monohydrate grown from aqueous solution, Journal of Physical Chemistry B, 104 (2000) 12256-12262.

[30] R.A. Visser, Supersaturation of alpha lactose in aqueous solutions in mutarotation equilibrium, Neth. Milk Dairy J., 36 (1982) 89-101.

[31] M.M. Reyhani, G.M. Parkinson, A comparison of secondary nuclei produced by contact of different growth faces of potash alum crystals under supersaturated solutions, J. Cryst. Growth, 166 (1996) 1068-1073.

[32] C.C. Sweeley, R. Bentley, M. Makita, W.W. Wells, Gas-loquid chromatography of trimethylsilyl derivatives of sugars and related compounds, Journal of American Chemical Society, 85 (1963) 2497-2507.

[33] T.D. Dincer, G.M. Parkinson, A.L. Rohl, M.I. Ogden, Crystallisation of $\alpha$-lactose monohydrate from DMSO solutions: Influence of $\beta$-lactose, J. Cryst. Growth, 205 (1999) 368-374.

[34] B.K. Butler , H. Zhang , M.R. Johns , D.L. Mackintosh , E.T. White The infl uence of growth rate dispersion in crystallisation in: CHEMECA , 25th Australian and New Zealand Chemical Engineer' s Conference and Exhibition Rotorua, New Zealand, 1997.

[35] T.D. Dincer, M.I. Ogden, G.M. Parkinson, Crystal growth mechanisms of the (010) face of alpha lactose monohydrate crystals, J. Cryst. Growth, 311 (2009) 2427-2432.

[36] A.A. Zekic, M.M. Mitrovic, S.M. Elezovic-Hadzic, D.A. Malivuk, Long-time growth rate changes of sodium chlorate, potassium dihydrogen phosphate and rochelle salt crystals independent of growth conditions, Industrial and Engineering Chemistry Research, 50 (2011) 8726-8733.

[37] A. van Kreveld, Growth rates of lactose crystals in solutions of stable anhydous alpha lactose, Neth. Milk Dairy J., 23 (1969) 158-275. 
[38] K. Sangwal, Growth Kinetics and Surface Morphology of Crystals Grown from Solution: Recent Observations and Their Interpretations, in: Progresses in Crystal Growth and Characterisation, Elsevier Sciences, 1998, pp. 163-248. 


\section{List of Figures}

Figure 1 Miller indices of a tomahawk shaped a-lactose monohydrate crystal [27]

Figure 2 In situ optical microscopy, showing growth of crystals for four days under experimental conditions

Figure 3 (a) The growth rates as a function of crystal size grown in situ at $30{ }^{\circ} \mathrm{C}$ and (s1) 0.55 and (b) Size and growth rates of crystals as a function of time for in situ, stagnant and bottle roller experiments

Figure 4 (a) L versus L/W and (b) Growth rates versus L/W ratios for the static (grown for 10 days for AFM imaging) and the bottle roller (BR) experiments

Figure 5 The (010) face of the small crystals (S1) and AFM image of the spiral (optical image on the top indicates the position of the spiral on the (010) face of the same crystal

Figure 6 The (010) face of a medium size crystal and the AFM image of the centre of the crystal

Figure 7 The (010) face of a large crystal (L1) and AFM image of the centre of the crystal

\section{List of Tables}

Table 1 Description of AFM imaged crystals 


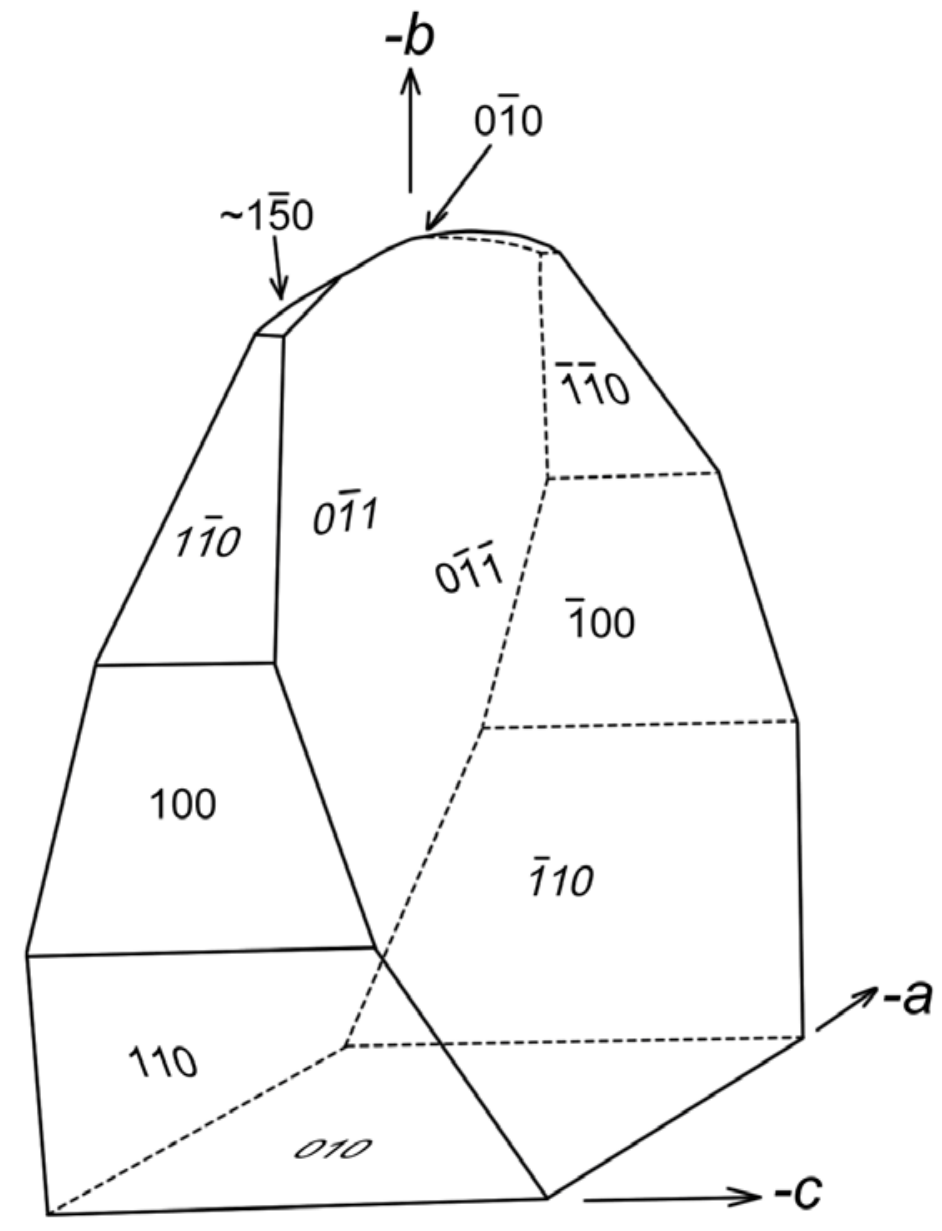

Figure 1 


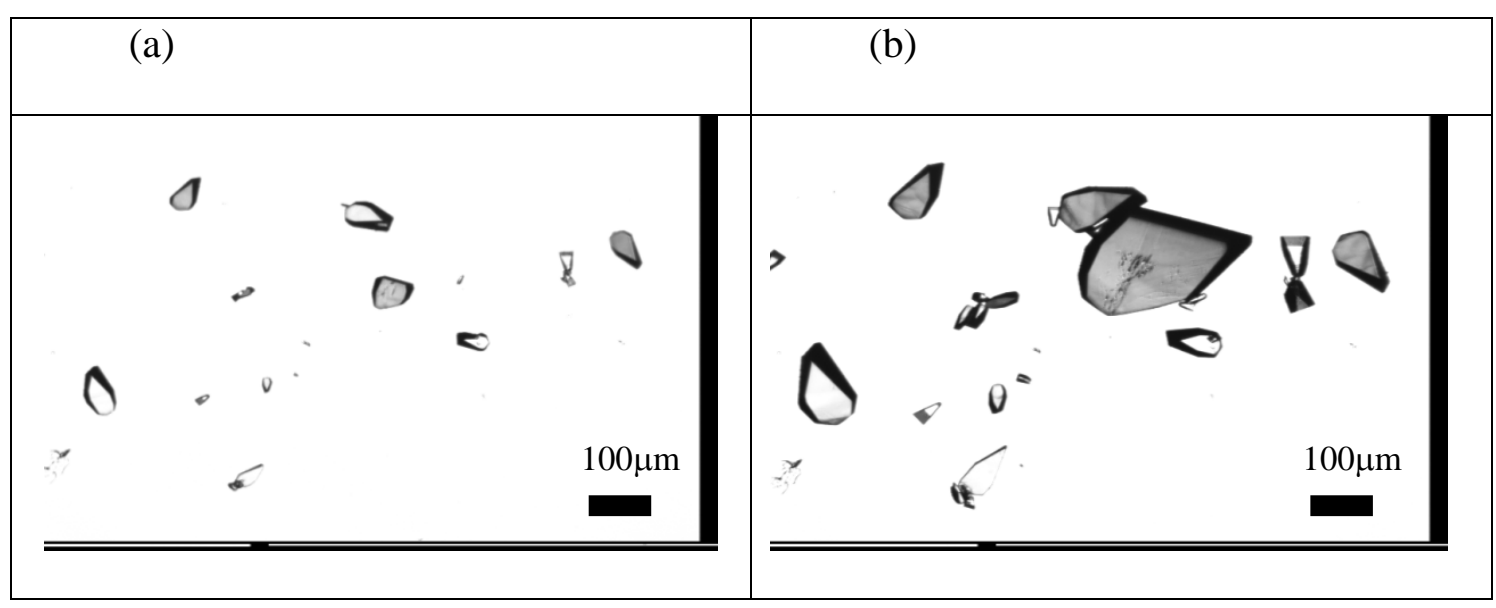

Figure 2 
(a)

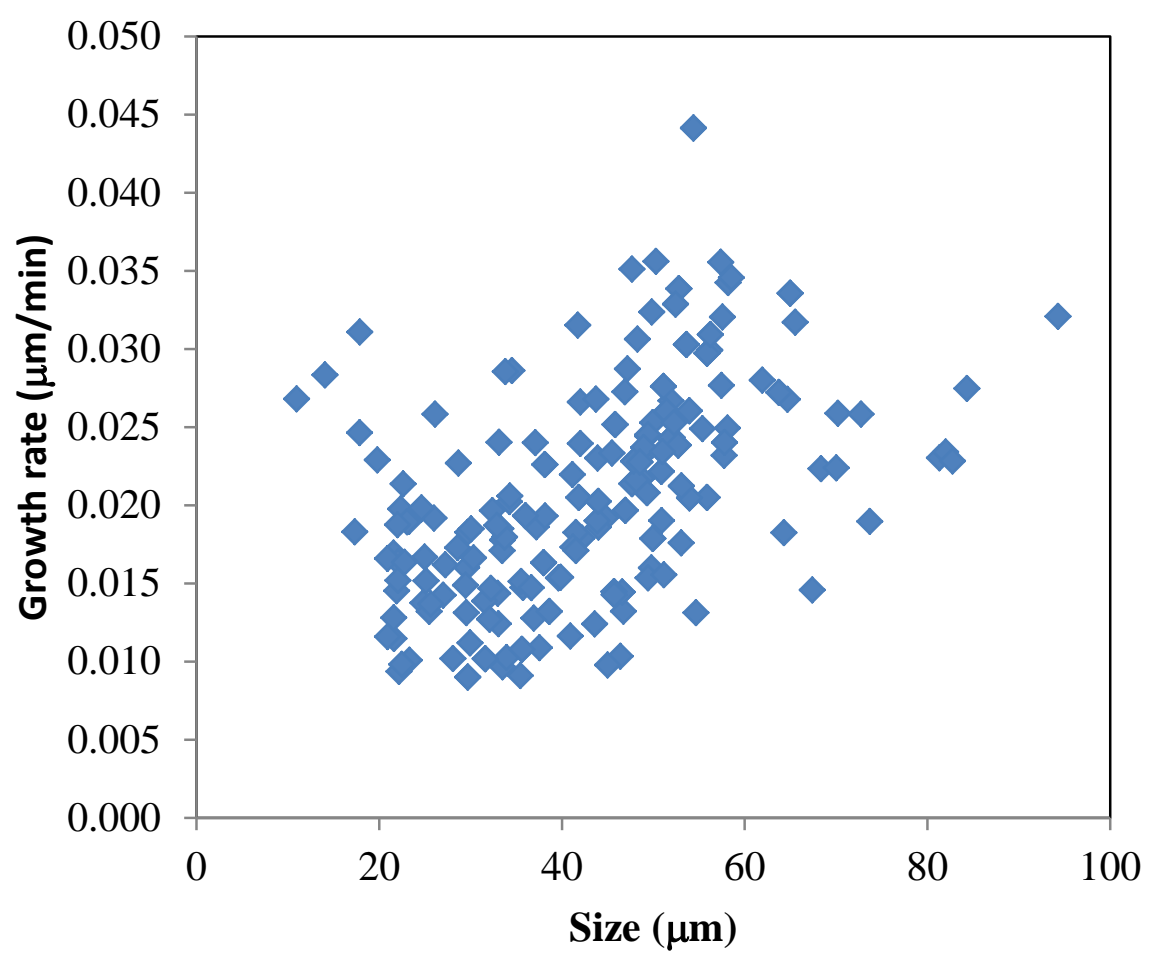

(b)

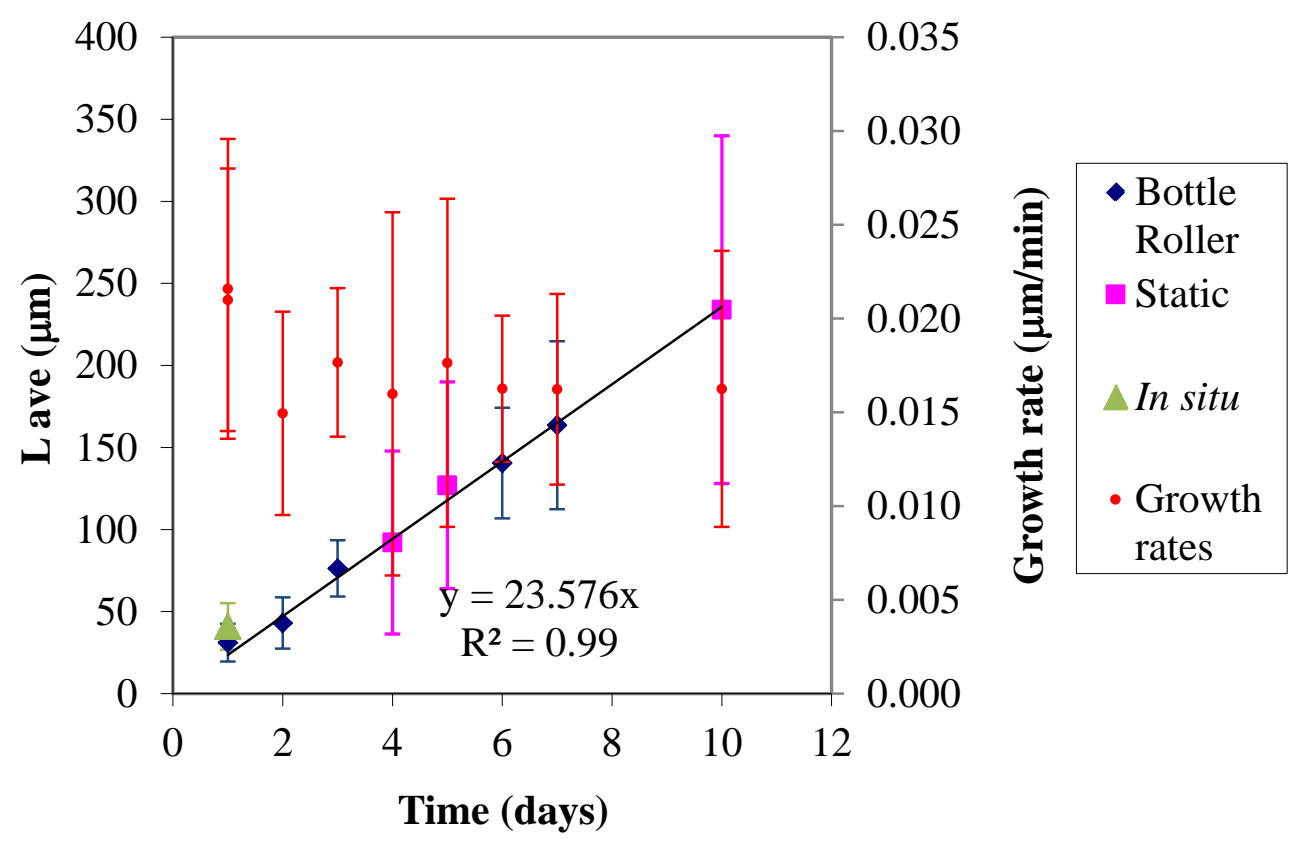

Figure 3 
(a)

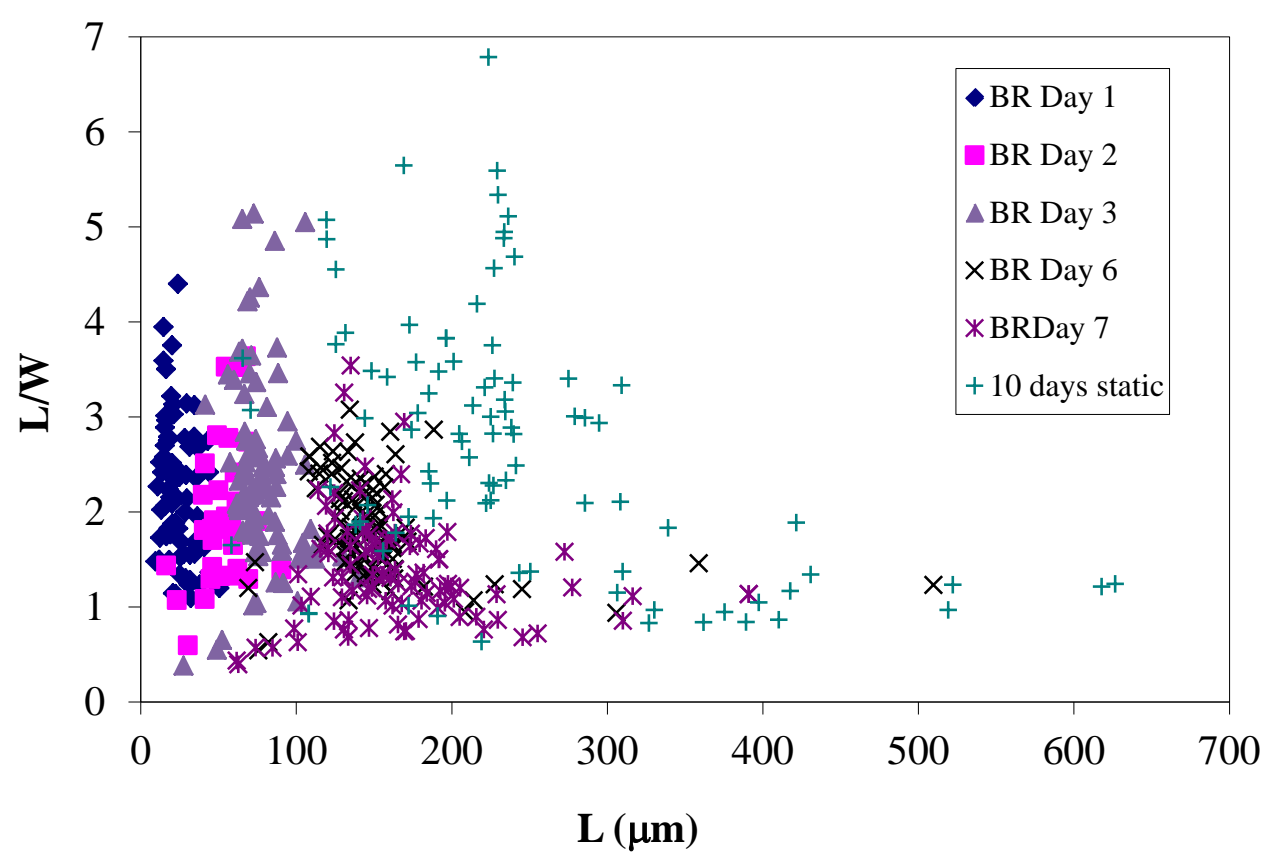

(b)

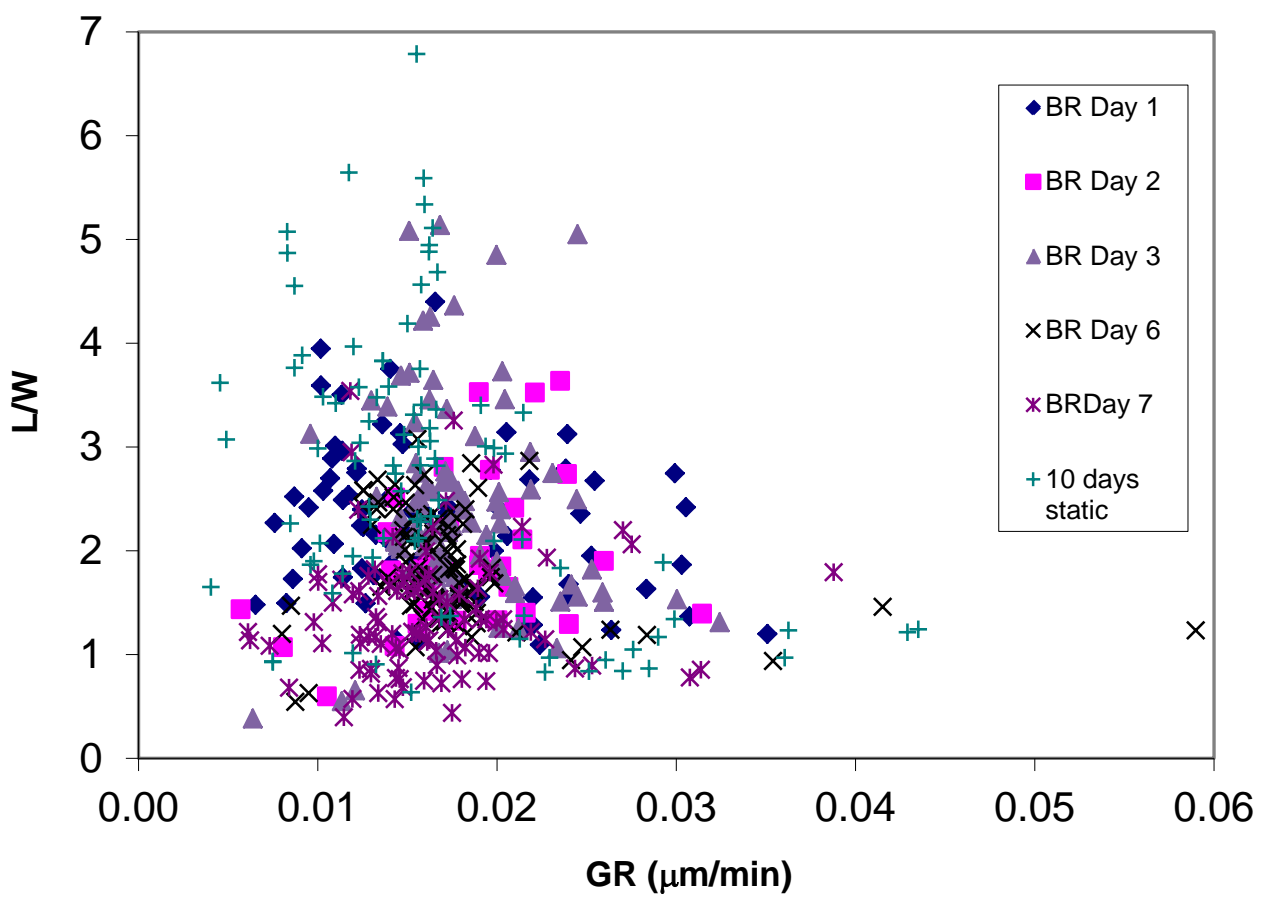

Figure 4 

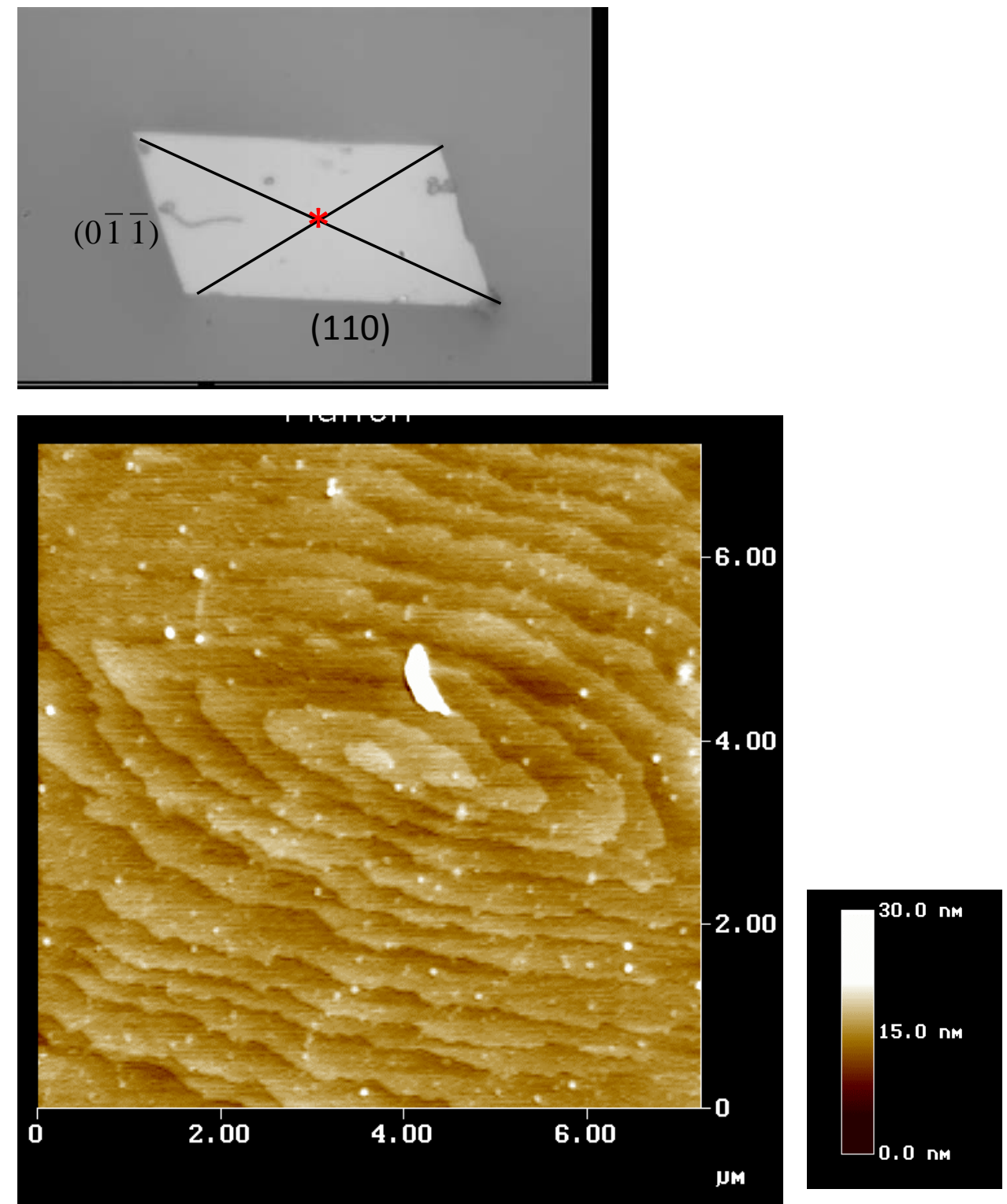

Figure 5 

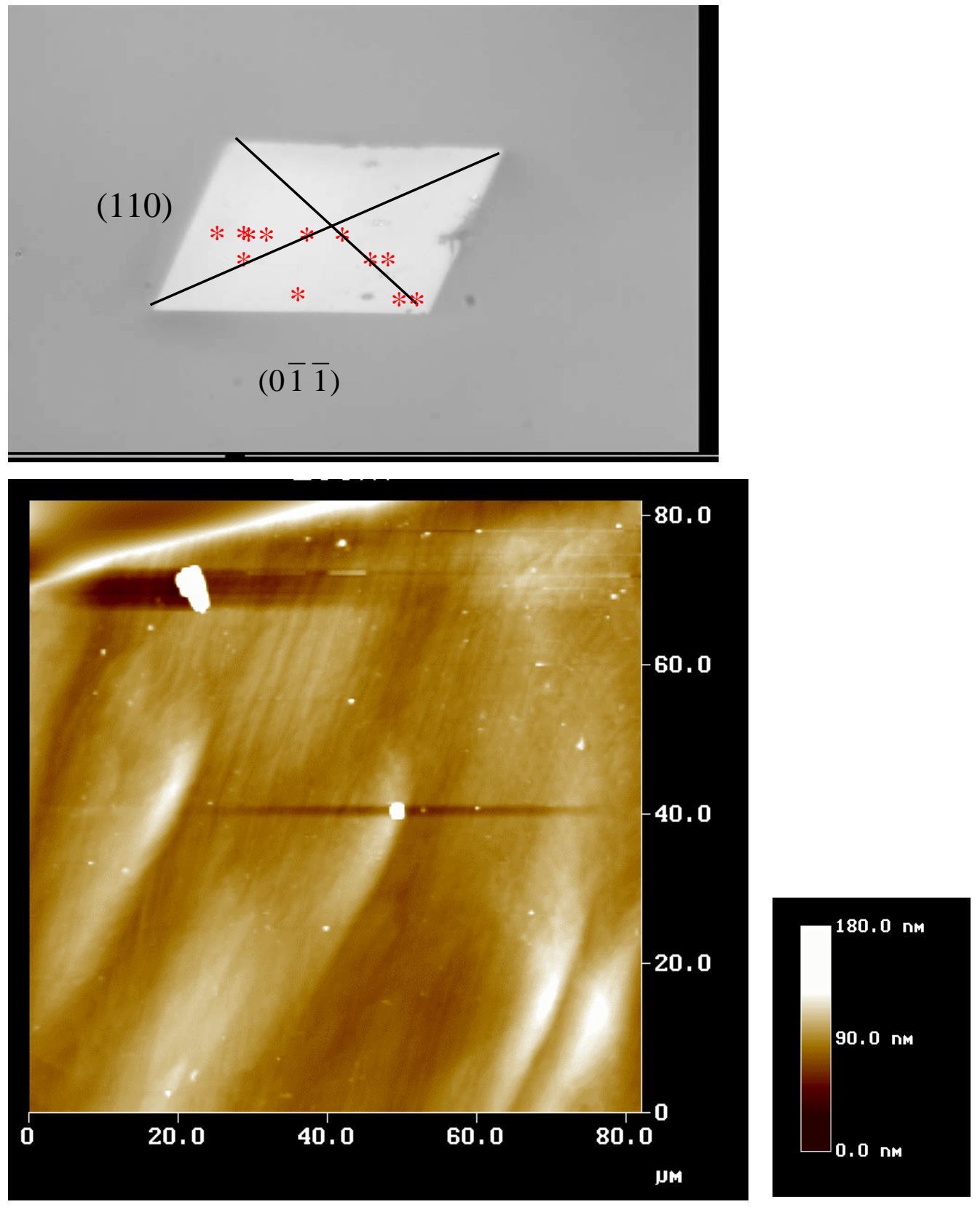

Figure 6 

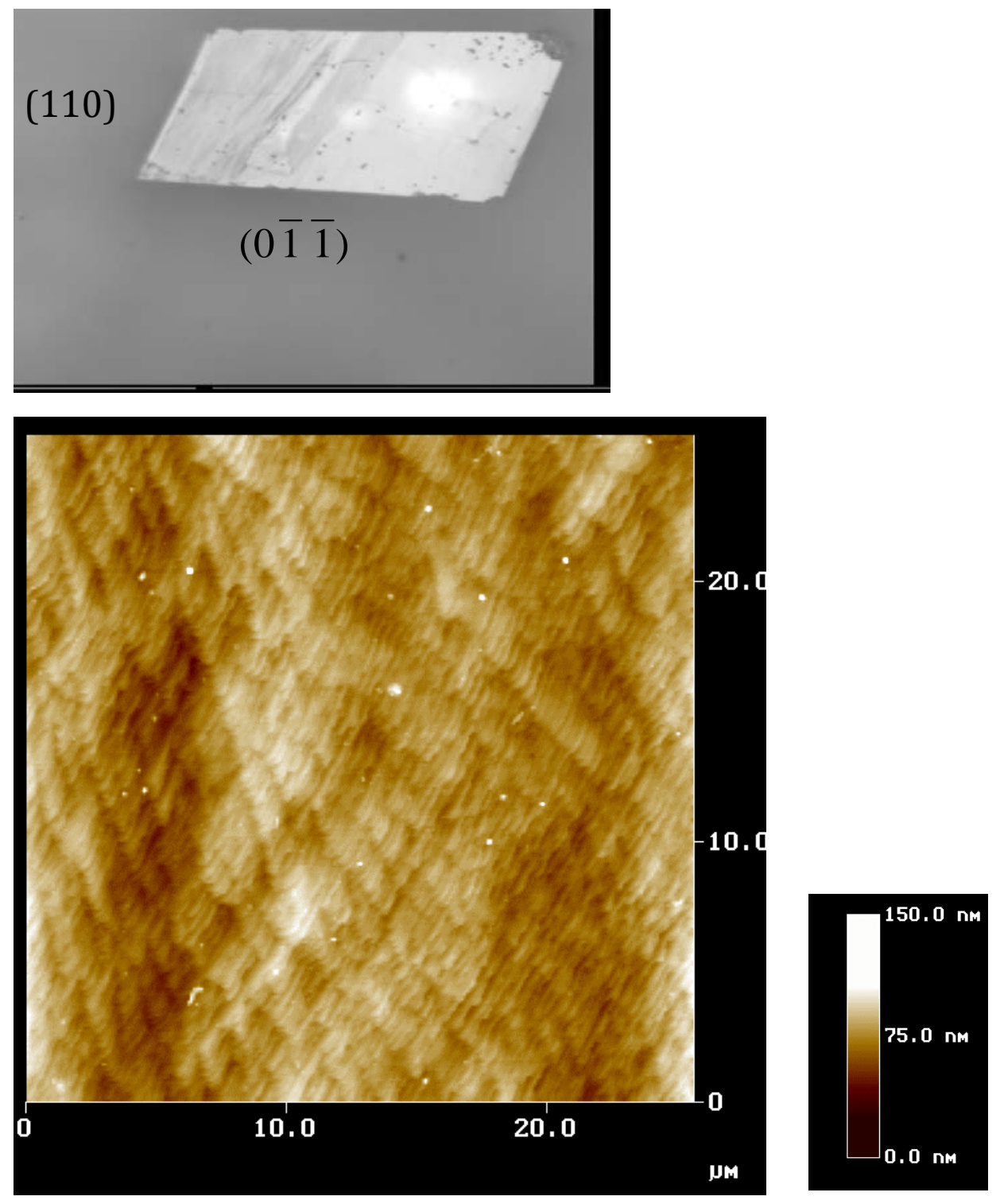

Figure 7 
Table 1

\begin{tabular}{|c|c|c|c|c|c|c|c|c|c|}
\hline Crystal & $\mathbf{L}$ & $\mathbf{W}$ & D & \# of & & Area & GR & $\mathbf{G R}_{\text {ave }}$ & \\
\hline ID & $(\mu \mathrm{m})$ & $(\mu \mathrm{m})$ & $(\mu \mathrm{m})$ & spirals & L/W & $\left(\mu \mathbf{m}^{2}\right)$ & $(\mu \mathrm{m} / \mathrm{min})$ & $(\mu \mathrm{m} / \mathrm{min})$ & $A_{\text {ave }}$ \\
\hline S1 & 166 & 48 & 92 & 1 & 3.5 & 4416 & 0.012 & & \\
\hline S3 & 144 & 48 & 80 & 1 & 3.0 & 3840 & 0.010 & 0.01 & 4107 \\
\hline S5 & 207 & 27.3 & 98.3 & 1 & 7.6 & 2684 & 0.014 & & \\
\hline $\mathrm{S} 2$ & 200 & 59 & 93 & 2 & 3.4 & 5487 & 0.014 & & \\
\hline M1 & 294 & 76 & 141 & 5 & 3.9 & 10716 & 0.020 & & \\
\hline M2 & 272 & 131 & 68 & 8 & 2.1 & 8908 & 0.019 & 0.02 & 13937 \\
\hline M3 & 268 & 172 & 129 & 6 & 1.6 & 22188 & 0.019 & & \\
\hline M4 & 241 & 52 & 110 & 2 & 4.6 & 5720 & 0.017 & & \\
\hline L1 & 460 & 431 & 189 & 0 & 1.1 & 81459 & 0.032 & & \\
\hline L2 & 510 & 543 & 279 & 0 & 0.9 & 151497 & 0.035 & 0.03 & 104561 \\
\hline L3 & 383 & 379 & 213 & 0 & 1.0 & 80727 & 0.027 & & \\
\hline
\end{tabular}

